with partners outside of one's household. We tried to elucidate motives and barriers for complying with sexual distancing among men who have sex with men (MSM) and the relation with social distancing.

Methods In this exploratory qualitative study we interviewed STI clinic visiting MSM during the first COVID-19 lock down using a semi-qualitative questionnaire from March-May 2020. We interviewed both men who complied and did not comply with the sexual distancing measures. The interviews were transcribed using verbatim transcription and analyzed using MAXQDA.

Results We included 18 non-compliers and 4 compliers. Motivations mentioned to comply with sexual distancing measures were: perceiving COVID-19 as a serious health threat, experiencing critically ill COVID-19 patients first hand, protecting someone dear from COVID-19, and being satisfied with social and/or sexual distancing measures. Barriers mentioned to comply with sexual distancing measures were: not being aware of the need for sexual distancing, being single, previously having had an active sex life, importance of the social aspect of sex, a strong urge for sex, having sex to reduce stress, being under the influence of alcohol or drugs, and not perceiving COVID-19 is a serious health threat.

Conclusion Our findings suggest that the information on sexual distancing needs to be made more explicit, accessible, understandable, customized and relatable to the key populations. This will improve effective measures and health advises for the current COVID-19 pandemic and future droplet borne outbreaks.

\section{P051 GENERAL PRACTITIONERS' (GPS') KNOWLEDGE OF AND ATTITUDES TO PRESCRIBING PRE-EXPOSURE PROPHYLAXIS FOR HIV (PREP): A PILOT STUDY}

'B Rai, 'S Ross*, 1,2D Richardson. 'Brighton and Sussex University Hospitals NHS Trust, Brighton, UK; ${ }^{2}$ Brighton and Sussex Medical School, Brighton, UK

\subsection{6/sextrans-2021-sti. 195}

Background In the UK PrEP is available only from sexual health services. The 2018 BHIVA/BASHH PrEP guidelines acknowledge the advantages of PrEP being delivered in sexual health services but raise concerns that this may restrict access for some people. Locally we have a large population of men who have sex with men (MSM) and provide PrEP to over 800, however many MSM and other people who would benefit from PrEP do not access sexual health services and may have more contact with primary care. We aimed to gain some insight into the knowledge and attitudes primary care have around PrEP to design an educational package.

Methods An online survey was circulated to GPs and trainee GPs working in an acute hospital trust.

Results Of the 12 respondents; $11(92 \%)$ were aware of PrEP, $6(50 \%)$ reported having been asked about PrEP by patients, $5(42 \%)$ had previously prescribed PrEP (as part of training in attachment in a sexual health clinic): 7(58\%) felt that some of their patients would benefit from PrEP, with the remaining $5(42 \%)$ being unsure. $3(25 \%)$ said they would prescribe PrEP for a patient at high risk of HIV in future, 4
(33\%) said they would not prescribe PrEP and 5(42\%) were unsure. Barriers to prescribing PrEP included unfamiliarity and uncertainty around which patients would benefit. 10 (83\%) respondents felt PrEP should only be prescribed in dedicated clinics or sexual health and $2(17 \%)$ suggested that PrEP should be available in primary care: $8(67 \%)$ felt training in prescribing and monitoring PrEP would be useful for primary care.

Conclusion Our educational package on PrEP in primary care needs to focus on identification of patients at high risk of HIV and increase the general knowledge of PrEP in primary care to facilitate clinical pathways and signposting to sexual health services.

\section{P052 PERFORMANCE OF THREE COMMERCIAL MOLECULAR DIAGNOSTIC ASSAYS FOR THE SIMULTANEOUS DETECTION OF MYCOPLASMA GENITALIUM AND MACROLIDE RESISTANCE}

${ }^{1} \mathrm{C}$ Le Roy, ${ }^{2} \mathrm{C}$ Bébéar, ${ }^{2} \mathrm{~S}$ Pereyre*.${ }^{1}$ University of Bordeaux, Bordeaux, France; ${ }^{2}$ National Reference Center for bacterial STI, Bordeaux university hospital, University of Bordeaux, Bordeaux, France

\subsection{6/sextrans-2021-sti.196}

Background The increasing frequency of macrolide resistance is an emerging issue in the treatment of Mycoplasma genitalium infection. Evaluation of new commercial kit detecting $\mathrm{M}$. genitalium and macrolide resistance-associated mutations is needed.

Methods We evaluated the performance and handling characteristics of the Allplex MG \& AziR (Seegene), the MacrolideR/MG ELITe MGB (ELITechGroup), and the ResistancePlus MG FleXible kits (SpeeDx-Cepheid) in comparison with an in-house real-time PCR and 23S rRNA gene sequencing used as reference. A total of 239 urogenital specimens $(135 \mathrm{M}$. genitalium-positive and $104 \mathrm{M}$. genitalium-negative specimens) collected between April and December 2019 at the French National Reference Center for bacterial Sexually Transmitted Infections were assessed.

Results The overall agreement for M. genitalium detection of the three commercial kits compared with the in-house realtime PCR was 94.6-97.6\%, and there was no significant difference. A total of 97 specimens were found M. genitaliumpositive with the three kits and were used to assess macrolide resistance detection. The clinical sensitivity for resistance detection was $74.5 \%$ (95\% confidence interval 61.7-84.2\%), 96.2\% (87.2-99.0\%), and 92.8\% (82.7-97.1\%) for the Allplex MG \& AziR, Macrolide-R/MG ELITe MGB, and ResistancePlusMG FleXible kits, respectively. The sensitivity of the Macrolide-R/MG ELITe MGB kit was significantly higher than that of the Allplex MG \& AziR kit. The clinical specificity for resistance detection of the three kits was 97.4-97.6\%. The random-access possibility, the input sample volume, and DNA extract availability for detecting resistance to other antibiotics may also influence the selection of a commercial kit by diagnostic laboratories.

Conclusion The three kits showed good performance for the detection of M. genitalium but the Allplex MG \& AziR kit showed lower sensitivity for detecting macrolide resistanceassociated mutations. 